\title{
CRITICAL EVALUATION OF FBD, PQ AND CPT CURRENT DECOMPOSITIONS FOR FOUR-WIRE CIRCUITS
}

\author{
Fernando P. Marafão $^{*} \quad$ Helmo K. M. Paredes ${ }^{\dagger} \quad$ Luiz C. P. da Silva $^{\dagger}$ \\ "Campus de Sorocaba, Unesp - Univ Estadual Paulista \\ Av. Três de Março, 511, Sorocaba, SP - Brazil - 18087-180. \\ ${ }^{\dagger}$ University of Campinas, School of Electrical and Computer Engineering \\ Av. Albert Einstein, 400, Campinas, SP - Brazil - 13083-970. \\ fmarafao@sorocaba.unesp.br \\ hmorales@dsee.fee.unicamp.br \\ lui@dsee.fee.unicamp.br
}

\begin{abstract}
This paper investigates the major similarities and discrepancies of three important current decompositions proposed for the interpretation of unbalanced and/or non linear three-phase four-wire circuits. The considered approaches were the so-called $F B D$ Theory, the $p q$-Theory and the CPT. Although the methods are based on different concepts, the results obtained under ideal conditions (sinusoidal and balanced signals) are very similar. The main differences appear in the presence of unbalanced and non linear load conditions. It will be demonstrated and discussed how the choice of the voltage referential and the return conductor impedance can influence in the resulting current components, as well as, the way of interpreting a power circuit with return conductor. Under linear unbalanced conditions, both FBD and pq-Theory suggest that the some current components contain a third-order harmonic. Besides, neither pq-Theory nor FBD method are able to provide accurate information for reactive current under unbalanced and distorted conditions, what seems to be done by means of the CPT.
\end{abstract}

Keywords - Current decomposition, FBD-theory, Homo-variables, Harmonics, $p q$-theory, Power theory, Unbalanced systems.

\section{INTRODUCTION}

The worldwide search for a generalized power theory, applicable for power systems under non-sinusoidal and/or unbalanced conditions, has mostly been motivated by the ever increasing of power electronic converters utilization. It points out the major requirement of improvement and adaptation of reactive/harmonic compensators technology and revenue metering techniques, especially on weak networks, such like those related to modern microgrids.

In this sense, numerous new power theories have been defined, and several of them are based on the frequency domain to describe suitable power and current terms under non-sinusoidal and unbalanced conditions [1]-[7]. On the other hand, giving special emphasis to instantaneous quantities, other important current decompositions and power definitions have been presented [8]-[17].

However, despite of the enormous efforts already spent, there is still no complete agreement on several current decompositions and related power definitions. Most of the

Manuscript received on 05/10/2009. Revised on 21/11/2009. Accepted by recommendation of the Editor Fernando L. M. Antunes. misunderstanding is probably caused since several authors usually had presented their contributions directed to a specific application, instead of discussing a general applicable power theory [18].

If there are still some doubts regarding to single-phase definitions [1],[5],[6],[8],[14],[23],[24],[41], the confusion and discrepancies are even greater in three-phase power systems, especially those with return or neutral conductor [5],[11],[18],[26],[28],[35]-[37],[41]. As it will be discussed, some of the misunderstanding can be explained in terms of the choice of the voltage referential and also in terms of the return conductor impedance.

Thus, considering just the time domain approaches, one could call attention to the proposals of Depenbrock $(F B D)$ [10],[11], Akagi et al. (pq-Theory) [12],[13] and Tenti et al. (CPT) [14],[15], which are strongly related to power conditioning applications.

Given that the $p q$-Theory is very well-known and accepted by the power electronics community, some authors tend to consider it a theoretical tool not only for active filter control [16],[19]-[21], but also for power properties' definitions and understanding [22],[23], regardless of all the misunderstanding about physical phenomena under non sinusoidal and unbalanced conditions [18],[24]-[26].

Willems [27] had already verified that the $p q$-Theory faces some conceptual problems. More recently, Depenbrock et al. [28] have investigated the original and modified pqTheory for three-phase four-wire systems, but it was Czarnecki [29],[30], who investigated how the properties of three-phase three-wire systems are described by means of the $p q$-Theory and discuss why such theory should not be used for understanding the power properties of the load instantaneously.

In the same way of the discussions of [31], for three-phase three-wire circuits, in this paper it will be demonstrated that the analysis of the physical phenomena based on the $F B D$ or on the $p q$-Theory can be also very confusing for three-phase four-wire circuits, since different effects caused by the loads are mixed up in the resulting decomposed currents.

Thus, next sections present a concise review of the investigated power theories and related current decompositions. Then, simulation results for three different load and PCC (Point of Common Coupling) conditions are discussed and compared in order to point out their major similarities and discrepancies. The discussions are not directed from the point of view of their applications, but as current decompositions for power properties elucidation. 


\section{THE $F B D$ THEORY}

The FBD (Fryze-Buchholz-Depenbrock) method is an extension of the Fryze [8] and Buchholz [9] theories, on which Depenbrock makes use of Fryze's current decomposition and Buchholz's instantaneous and RMS collective values for the definition of new current decompositions. Such currents were also applied to the calculation of novel power components and for the proposition of compensation strategies [10],[11].

According to the author, the $F B D$-method can be applied in any multiphase power circuit, which can be represented by a uniform circuit on which none of the conductors is treated as an especial conductor. In this uniform circuit, the voltages in the m-terminals are referred to a virtual star point "**". The single prerequisite is that Kirchhoff's laws must be valid for the voltages and currents at the terminals [11]. Here, it is important to point out that the measured voltages to the virtual star point may not represent the instantaneous values of the voltages over the load terminals [32], especially under unbalanced and non-sinusoidal conditions.

Thus, considering multiphase power circuits, the $F B D$ method uses multidimensional voltage and current vectors $\left(\boldsymbol{v}_{*}, \boldsymbol{i}\right)$ and their instantaneous collective values $\left(v_{\Sigma}, i_{\Sigma}\right)$, defined respectively as:

$$
\begin{gathered}
\boldsymbol{v}_{*}=\left[\begin{array}{c}
v_{a *} \\
v_{b *} \\
v_{c *} \\
\vdots \\
v_{m *}
\end{array}\right], \quad \boldsymbol{i}=\left[\begin{array}{c}
i_{a} \\
i_{b} \\
i_{c} \\
\vdots \\
i_{m}
\end{array}\right], \\
v_{\Sigma}=\sqrt{\sum_{\mu=1}^{m} v_{\mu *}^{2},} \quad i_{\Sigma}=\sqrt{\sum_{\mu=1}^{m} i_{\mu}^{2},}
\end{gathered}
$$

where, " $m$ " indicates the number of conductors and bold letters indicate vector representation. Thus, the collective instantaneous power results from the inner (dot) product:

$$
p_{\Sigma}=\boldsymbol{v}_{*}{ }^{t} \cdot \boldsymbol{i},
$$

where $\left(x^{t}\right)$ was used here to indicate the transpose.

Under periodic conditions the collective RMS value of the currents and of the voltages can be calculated as:

$$
I_{\Sigma}=\sqrt{\frac{1}{T} \int_{0}^{T} i_{\Sigma}^{2} d t}, \quad V_{\Sigma}=\sqrt{\frac{1}{T} \int_{0}^{T} v_{\Sigma}^{2} d t},
$$

and the collective "active" power results from:

$$
P_{\Sigma}=\frac{1}{T} \int_{0}^{T} p_{\Sigma} d t
$$

Thus, according to $F B D$-method, the instantaneous current through each phase of the system $\left(i_{\mu}\right)$ is decomposed in some components, proportional and orthogonal to the voltages:

Active currents $\left(i_{a \mu}\right)$ : responsible for the transference of average energy to the load. These definitions are generally valid under periodical condition and $i_{a \mu}$ is responsible for the same active power as the current $i_{\mu}$.

$$
i_{a \mu}=\frac{P_{\Sigma}}{V_{\Sigma}^{2}} v_{\mu *}=G_{a} v_{\mu *} .
$$

Nonactive currents $\left(i_{n \mu}\right)$ : associated to any type of disturbance and oscillations that affect the instantaneous power, but do not transfer average energy to the load.

$$
i_{n \mu}=i_{\mu}-i_{a \mu}
$$

Power currents $\left(i_{p \mu}\right)$ : responsible for the instantaneous power, including possible oscillations related with harmonic and unbalances.

$$
i_{p \mu}=\frac{p_{\Sigma}}{v_{\Sigma}^{2}} v_{\mu *}=G_{p} v_{\mu *}
$$

Powerless currents $\left(i_{z \mu}\right)$ : they do not contribute for the energy conveyance and can be compensated instantaneously without the necessity of energy storage elements.

$$
i_{z \mu}=i_{\mu}-i_{p \mu}
$$

Variation currents $\left(i_{v \mu}\right)$ : responsible for the oscillation of the instantaneous equivalent conductance $G_{p}$ around its average value $G_{a}$, or also, variations of $p_{\Sigma}$ around $P_{\Sigma}$.

$$
i_{v \mu}=i_{p \mu}-i_{a \mu}=i_{n \mu}-i_{z \mu}
$$

Thus, the orthogonal currents decompositions proposed by FBD results that:

$$
\left\|i_{\mu}\right\|^{2}=\left\|i_{a \mu}\right\|^{2}+\left\|i_{n \mu}\right\|^{2}=\left\|i_{a \mu}\right\|^{2}+\left\|i_{v \mu}\right\|^{2}+\left\|i_{z \mu}\right\|^{2} .
$$

\section{THE $P Q$-THEORY}

The instantaneous power theory proposed by Akagi et al. [12] is usually known as $p q$-Theory. This theory is based on the Clarke's transformation of voltages and currents from $(a, b, c)$-frame to $(\alpha, \beta, 0)$ orthogonal coordinates [39].

The pq-Theory describes the power properties of threephase three-wire systems by means of two main instantaneous power components: the instantaneous real power $p$, and the instantaneous imaginary power $q$. The proposal could also be applied for three-phase four-wire system by introducing the instantaneous zero-sequence power $p_{0}[13],[22]$. This theory was originally proposed as a mathematical tool, directed for the control of active power filters and this was one of the most important motivations for its great dissemination during the last two decades. It is worth mention that Akagi et al. spread the concept of reactive compensation without energy storage elements.

Thus, the phase voltages in the $\alpha, \beta$ and 0 coordinates have the form:

$$
\left[\begin{array}{l}
v_{0} \\
v_{\alpha} \\
v_{\beta}
\end{array}\right]=\sqrt{\frac{2}{3}}\left[\begin{array}{ccc}
\frac{1}{\sqrt{2}} & \frac{1}{\sqrt{2}} & \frac{1}{\sqrt{2}} \\
1 & -\frac{1}{2} & -\frac{1}{2} \\
0 & \frac{\sqrt{3}}{2} & -\frac{\sqrt{3}}{2}
\end{array}\right]\left[\begin{array}{l}
v_{a} \\
v_{b} \\
v_{c}
\end{array}\right]=C_{\mathbf{1}}\left[\begin{array}{l}
v_{a} \\
v_{b} \\
v_{c}
\end{array}\right] .
$$


Similarly, the instantaneous currents $\left(i_{a}, i_{b}\right.$ and $\left.i_{c}\right)$ can be transformed to the $\alpha, \beta$ and 0 coordinates. Note that in the case of three-wire systems, the measured phase voltages are referred to a virtual star point (as in the $F B D$ ) and with fourwire systems, the voltages are referred to the return conductor.

Therefore, considering four-wire circuits, the authors define three instantaneous power components as:

$$
\left[\begin{array}{c}
p_{0} \\
p \\
q
\end{array}\right]=\sqrt{\frac{2}{3}}\left[\begin{array}{ccc}
v_{0} & 0 & 0 \\
0 & v_{\alpha} & v_{\beta} \\
0 & v_{\beta} & -v_{\alpha}
\end{array}\right]\left[\begin{array}{c}
i_{0} \\
i_{\alpha} \\
i_{\beta}
\end{array}\right]
$$

The sum of $p_{0}$ and $p$ results in the traditional instantaneous power of three-phase systems:

$$
p_{3 \phi}=p+p_{0}=v_{\alpha} i_{\alpha}+v_{\beta} i_{\beta}+v_{0} i_{0} .
$$

Then, the authors introduced the concept of the instantaneous imaginary power " $q$ ", defined as:

$$
q=v_{\beta} i_{\alpha}-v_{\alpha} i_{\beta} \text {. }
$$

Using these two instantaneous power, $p$ and $q$, the orthogonal currents $i_{\alpha}$ and $i_{\beta}$ can be decomposed into instantaneous active $\left(i_{\alpha p}\right.$ and $\left.i_{\beta p}\right)$ and reactive $\left(i_{\alpha q}\right.$ and $\left.i_{\beta q}\right)$ currents, as follows:

$$
\begin{gathered}
i_{\alpha p}=\frac{v_{\alpha}}{v_{\alpha \beta}^{2}} p ; i_{\beta p}=\frac{v_{\beta}}{v_{\alpha \beta}^{2}} p, \\
i_{\alpha q}=\frac{v_{\beta}}{v_{\alpha \beta}^{2}} q ; i_{\beta q}=-\frac{v_{\alpha}}{v_{\alpha \beta}^{2}} q,
\end{gathered}
$$

where $v_{\alpha \beta}^{2}=v_{\alpha}^{2}+v_{\beta}^{2}$.

Accordingly, the instantaneous zero-phase sequence, active and reactive currents can be calculated in the original coordinates, by means of the inverse Clarke transformation:

$$
\begin{gathered}
{\left[\begin{array}{l}
i_{a 0} \\
i_{b 0} \\
i_{c 0}
\end{array}\right]=\sqrt{\frac{2}{3}}\left[\begin{array}{ccc}
\frac{1}{\sqrt{2}} & 1 & 0 \\
\frac{1}{\sqrt{2}} & -\frac{1}{2} & \frac{\sqrt{3}}{2} \\
\frac{1}{\sqrt{2}} & -\frac{1}{2} & -\frac{\sqrt{3}}{2}
\end{array}\right]\left[\begin{array}{l}
i_{0} \\
0 \\
0
\end{array}\right]=\mathbf{C}_{2}\left[\begin{array}{c}
i_{0} \\
0 \\
0
\end{array}\right],} \\
{\left[\begin{array}{l}
i_{a p} \\
i_{b p} \\
i_{c p}
\end{array}\right]=\mathbf{C}_{2}\left[\begin{array}{c}
0 \\
i_{\alpha p} \\
i_{\beta p}
\end{array}\right] \text { and }\left[\begin{array}{l}
i_{a q} \\
i_{b q} \\
i_{c q}
\end{array}\right]=\mathbf{C}_{2}\left[\begin{array}{c}
0 \\
i_{\alpha q} \\
i_{\beta q}
\end{array}\right] .}
\end{gathered}
$$

So, the instantaneous three-phase currents ( $a, b$ and $c)$ can be decomposed on the following components:

$$
\left[\begin{array}{l}
i_{a} \\
i_{b} \\
i_{c}
\end{array}\right]=\left[\begin{array}{l}
i_{a 0} \\
i_{b 0} \\
i_{c 0}
\end{array}\right]+\left[\begin{array}{l}
i_{a p} \\
i_{b p} \\
i_{c p}
\end{array}\right]+\left[\begin{array}{l}
i_{a q} \\
i_{b q} \\
i_{c q}
\end{array}\right]
$$

According to [16], the powers of (13) could also be decomposed into:

$$
p=\bar{p}+\tilde{p}, \quad q=\bar{q}+\tilde{q},
$$

where $\bar{p}$ and $\tilde{p}$ represent the average and oscillating component of $p$; and where $\bar{q}$ and $\tilde{q}$ represent the average and oscillating component of $q$. It was assumed that the oscillating parts of $p$ and $q$ are related to the occurrence of unbalanced and/or distorted voltages and currents.

Consequently, the instantaneous active current can also be decomposed into average $(\bar{x})$ and oscillating $(\tilde{x})$ component.

$$
\begin{aligned}
& i_{\alpha p}=\frac{v_{\alpha}}{v_{\alpha}^{2}+v_{\beta}^{2}} \bar{p}+\frac{v_{\alpha}}{v_{\alpha}^{2}+v_{\beta}^{2}} \tilde{p}=i_{\alpha \bar{p}}+i_{\alpha \tilde{p}} \\
& i_{\beta p}=\frac{v_{\beta}}{v_{\alpha}^{2}+v_{\beta}^{2}} \bar{p}+\frac{v_{\beta}}{v_{\alpha}^{2}+v_{\beta}^{2}} \tilde{p}=i_{\beta \bar{p}}+i_{\beta \tilde{p}} .
\end{aligned}
$$

Resulting in the $(a, b, c)$ coordinates:

$$
\left[\begin{array}{c}
i_{a p} \\
i_{b p} \\
i_{c p}
\end{array}\right]=\mathbf{C}_{\mathbf{2}}\left[\begin{array}{c}
0 \\
i_{\alpha \bar{p}} \\
i_{\beta \bar{p}}
\end{array}\right]+\mathbf{C}_{\mathbf{2}}\left[\begin{array}{c}
0 \\
i_{\alpha \tilde{p}} \\
i_{\beta \tilde{p}}
\end{array}\right]=\left[\begin{array}{c}
i_{a \bar{p}} \\
i_{b \bar{p}} \\
i_{c \bar{p}}
\end{array}\right]+\left[\begin{array}{c}
i_{a \tilde{p}} \\
i_{b \tilde{p}} \\
i_{c \tilde{p}}
\end{array}\right] .
$$

Finally, the instantaneous phase currents yield:

$$
\begin{aligned}
{\left[\begin{array}{l}
i_{a} \\
i_{b} \\
i_{c}
\end{array}\right]=} & {\left[\begin{array}{l}
i_{a p} \\
i_{b p} \\
i_{c p}
\end{array}\right]+\left[\begin{array}{l}
i_{a q} \\
i_{b q} \\
i_{c q}
\end{array}\right]+\left[\begin{array}{l}
i_{a 0} \\
i_{b 0} \\
i_{c 0}
\end{array}\right]=} \\
& {\left[\begin{array}{l}
i_{a \bar{p}} \\
i_{b \bar{p}} \\
i_{c \bar{p}}
\end{array}\right]+\left[\begin{array}{l}
i_{a \tilde{p}} \\
i_{b \tilde{p}} \\
i_{c \tilde{p}}
\end{array}\right]+\left[\begin{array}{l}
i_{a q} \\
i_{b q} \\
i_{c q}
\end{array}\right]+\left[\begin{array}{l}
i_{a 0} \\
i_{b 0} \\
i_{c 0}
\end{array}\right] . }
\end{aligned}
$$

However, neither the interpretation of these phase current components, nor their association with specific physical phenomena was directly treated by the authors of the $p q$ Theory.

\section{THE CPT FRAMEWORK}

The third considered approach (Conservative Power Theory, $C P T$ ) was recently proposed by Tenti et al. [14] and it is based on the definition of instantaneous complex power under non-sinusoidal conditions and it represents an extension of the usual complex power, defined for sinusoidal conditions. Even though detailed discussion has been directed to single-phase systems, this theory is also easily extended to multiphase systems [33].

The authors had introduced the so-called homo-variables (integral and derivate) which can be defined under periodic conditions and are homogeneous to the current, voltage and power terms. Since homo-voltages and homo-currents satisfy the Kirchhoff's Laws, the corresponding homo-powers are conservative in any electric network, what allows introducing the concept of conservation of the complex power under nonsinusoidal conditions. In addition, a current decomposition was proposed, on which every term is related to a specific physical phenomenon (power absorption $P$, energy storage $Q$, voltage and current distortion $D$ ). Moreover, it has been discussed its application to harmonic and reactive compensation, for local or distributed devices [15],[33].

Assuming multidimensional systems, the following definitions make use of the same symbols applied to the $F B D$-method (bold variables to vector representation and the index " $\mu$ " for each m-phase variable). Regarding to the voltage referential, the CPT authors suggest using the return conductor in case of its existence (differently from the $F B D$ approach) and the virtual point unless [33]. Thus, the homointegrals of the voltages and currents are defined as: 


$$
\begin{aligned}
& \hat{v}_{\mu}(t)=\omega\left(v_{\mu_{f}}(t)-\bar{v}_{\mu_{f}}\right), \\
& \hat{\imath}_{\mu}(t)=\omega\left(i_{\mu_{f}}(t)-\bar{\imath}_{\mu_{f}}\right),
\end{aligned}
$$

where: $v_{\mu \rho}(t)=\int_{0}^{T} v_{\mu}(\tau) d \tau, \quad i_{\mu \rho}(t)=\int_{0}^{T} i_{\mu}(\tau) d \tau$, are time integrals of voltages $v_{\mu}$ and currents $i_{\mu}$, and $\bar{v}_{\mu \int}, \bar{i}_{\mu \int}$ are the average value of each $v_{\mu}$ and $i_{\mu \rho}$, over period $T$.

Note that $\hat{\boldsymbol{v}}$ and $\hat{\boldsymbol{\imath}}$ are dimensionally homogeneous to voltage and current respectively. It means that the operation of integration does not influence the resulting amplitudes, since they are multiplied by the angular frequency.

Likewise, the homo-derivatives of the voltages and currents are given by:

$$
\check{v}_{\mu}(t)=\frac{1}{\omega} \frac{d v_{\mu}(t)}{d t} ; \quad \check{\iota}_{\mu}(t)=\frac{1}{\omega} \frac{d i_{\mu}(t)}{d t} .
$$

As well as $\widehat{\boldsymbol{v}}$ and $\hat{\boldsymbol{\imath}}$ the variables $\breve{\boldsymbol{v}}$ and $\check{\boldsymbol{\imath}}$ are also dimensionally homogeneous to the original voltages and currents, respectively. In this case the derivative is multiplied by the inverse of the angular frequency.

Considering periodical quantities, with period $T$ and fundamental frequency $\omega=2 \pi / T$, it is well-known that the internal product of voltage and current vectors is defined as:

$$
\langle\boldsymbol{v}, \boldsymbol{i}\rangle=\langle\boldsymbol{i}, \boldsymbol{v}\rangle=\frac{1}{T} \int_{0}^{T} \boldsymbol{v}(t) \cdot \boldsymbol{i}(t) d t,
$$

and in the same way, the voltage and current norms are:

$$
\begin{aligned}
\|\boldsymbol{v}\| & =\sqrt{\langle\boldsymbol{v}, \boldsymbol{v}\rangle}, \\
\|\boldsymbol{i}\| & =\sqrt{\langle\boldsymbol{i}, \boldsymbol{i}\rangle},
\end{aligned}
$$

which results equal to the RMS collective values from the $F B D$-method $\left(V_{\Sigma}, I_{\Sigma}\right)$. For single-phase circuits or considering each phase of an $\mathrm{m}$-dimensional system:

$\left\|v_{\mu}\right\|=\sqrt{\frac{1}{T} \int_{0}^{T} v_{\mu}^{2}(t) d t}=V_{\mu}, \quad\left\|i_{\mu}\right\|=\sqrt{\frac{1}{T} \int_{0}^{T} i_{\mu}^{2}(t) d t}=I_{\mu}$, yields the RMS phase voltages and currents.

Under the assumption of periodic behavior, the following quantities have been defined, which are valid for both sinusoidal and distorted, balanced or unbalanced conditions:

Active power: that represents the conveyed average power. This definition is identical to the conventional active power (Steinmetz, Budeanu, Fryze, Buchholz, Depenbrock, Czarnecki, STD-1459):

$$
P=\langle\boldsymbol{v}, \boldsymbol{i}\rangle=\frac{1}{T} \int_{0}^{T} \boldsymbol{v}(t) \cdot \boldsymbol{i}(t) d t .
$$

Reactive power: that represents the average energy stored in the network and was defined as:

$$
Q=\langle\widehat{\boldsymbol{v}}, \boldsymbol{i}\rangle=\frac{1}{T} \int_{0}^{T} \widehat{\boldsymbol{v}}(t) \cdot \boldsymbol{i}(t) d t .
$$

The physical meaning of this and other power terms are widely discussed in [14]. Following, the total currents are split into some parcels, regarding to their association to the power terms. The active current is the minimum current (i.e., with minimum norm) conveying active power $P$ to the load and it is defined as:

$$
i_{a \mu}=\frac{P}{\|v\|^{2}} \cdot v_{\mu}=G_{e} \cdot v_{\mu} .
$$

The reactive current is the minimum current transferring reactive power $Q$, and it is related to the average energy being exchanged through the circuit:

$$
i_{r \mu}=\frac{Q}{\|\hat{v}\|^{2}} \hat{v}_{\mu}=B_{e} \hat{v}_{\mu}
$$

Both the active and reactive currents have an explicit physical meaning. They are associated with the presence of the active and reactive powers, $P$ and $Q$, and are related to the load average equivalent conductance $G_{e}$, and susceptance, $B_{e}$.

The void current is the remaining current (residual term), since it does not convey active $P$ nor reactive $Q$ power:

$$
i_{v \mu}=i_{\mu}-i_{a \mu}-i_{r \mu}
$$

According to the original CPT proposal [14], the void currents may exist only in presence of current distortion, what it's exact in case of single-phase systems. However it will be demonstrated in the following simulations that it is also influenced by current unbalances, in case of multi-phase circuits. Further details of each current component and its physical meaning can be found in [14]. By definition, all current terms are orthogonal:

$$
\|\boldsymbol{i}\|^{2}=\left\|\boldsymbol{i}_{\boldsymbol{a}}\right\|^{2}+\left\|\boldsymbol{i}_{\boldsymbol{r}}\right\|^{2}+\left\|\boldsymbol{i}_{v}\right\|^{2} .
$$

\section{SIMULATION RESULTS: COMPARISON AND DISCUSSION}

Assuming just the three-phase four-wire power circuits, next sections demonstrate the main similarities and differences among the previous discussed proposals. The main goal is to compare the resulting current components by means of each method. In order to do that, three different conditions were simulated and analyzed by means of the PSIM software.

In order to make easier the comparisons, the following under scripts were applied: $F B D, p q, C P T$ for the considered approaches and $\mu$ indicating phase-variables.

\section{A. Case I: Unbalanced resistive load - small line impedance}

Figure 1 shows the power circuits for Case I. Note that the voltage referential changes accordingly to the evaluated proposal (Figs. 1-a and 1-b). Table 1 depicts the values of grid voltages, line impedance and load phase resistances.

As expected, the load unbalance causes current flowing by the return conductor (n-neutral) and accordingly to the $F B D$ method, such conductor should be treated as equal in an $\mathrm{m}$ conductor polyphase methodology [10],[11]. Thus, assuming a three-phase four-wire $(m=4)$ circuit, four voltages and four currents are measured as shown in Fig. 1-a. The other two methods are based on $m-1$ voltage and current measures [34].

Figures 2-4 show the measured PCC voltages $(v)$ and currents $(i)$ and the resulting current components from each method. 


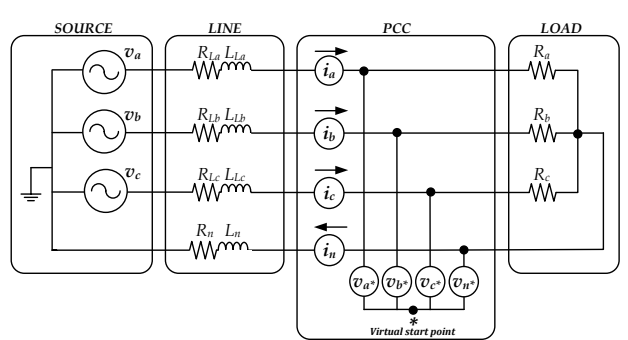

a) Voltages measured to a virtual star point $(*)$ : $F B D$-Method

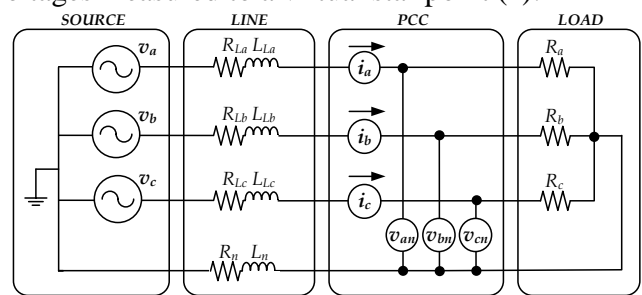

b) Voltages measured to the return conductor: $p q$-Theory and $C P T$.

Fig. 1. Power circuits for Case I - unbalanced resistive load.

TABLE I

Voltages and impedances for Case I.

\begin{tabular}{cll}
\hline \multicolumn{1}{c}{ Source } & \multicolumn{1}{c}{ Line } & Load (Y) \\
\hline $\mathrm{V}_{\mathrm{a}}=127 \angle 0^{\circ}$ Vrms & $\mathrm{R}_{\mathrm{La}}=1 \mathrm{~m} \Omega \quad \mathrm{L}_{\mathrm{La}}=10 \mu \mathrm{H}$ & $\mathrm{R}_{\mathrm{a}}=9,3405 \Omega$ \\
\hline $\mathrm{V}_{\mathrm{b}}=127 \angle-120^{\circ} \mathrm{Vrms}$ & $\mathrm{R}_{\mathrm{Lb}}=1 \mathrm{~m} \Omega \quad \mathrm{L}_{\mathrm{Lb}}=10 \mu \mathrm{H}$ & $\mathrm{R}_{\mathrm{b}}=6,2270 \Omega$ \\
\hline $\mathrm{V}_{\mathrm{c}}=127 \angle 120^{\circ} \mathrm{Vrms}$ & $\mathrm{R}_{\mathrm{Lc}}=1 \mathrm{~m} \Omega \quad \mathrm{L}_{\mathrm{Lc}}=10 \mu \mathrm{H}$ & $\mathrm{R}_{\mathrm{c}}=3,1135 \Omega$ \\
\hline \multicolumn{3}{c}{$\mathrm{R}_{\mathrm{n}}=1 \mathrm{~m} \Omega \mathrm{L}_{\mathrm{n}}=10 \mu \mathrm{H}$} \\
\hline
\end{tabular}
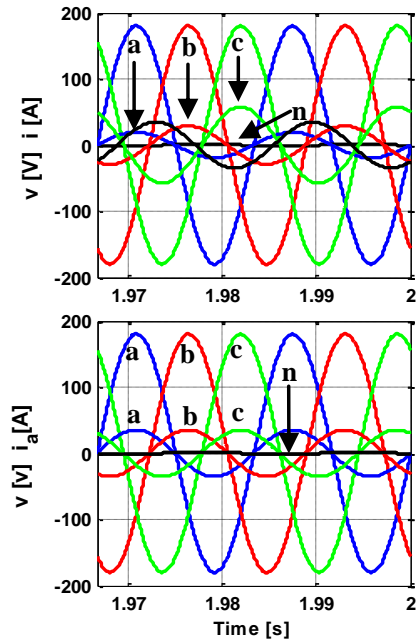

Fig. 2. Measured PCC voltages and currents and resulting components for Case I - FBD-Method
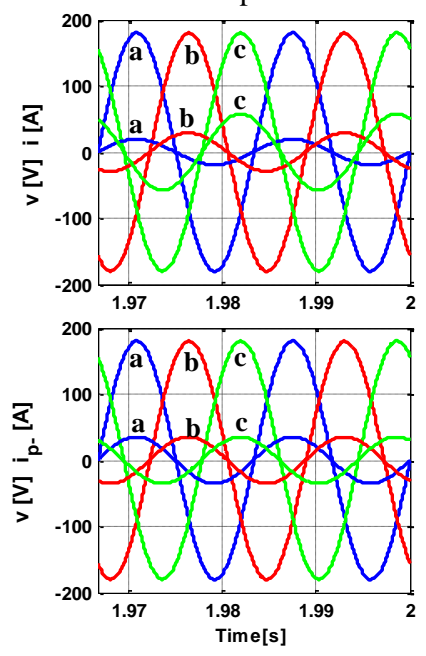

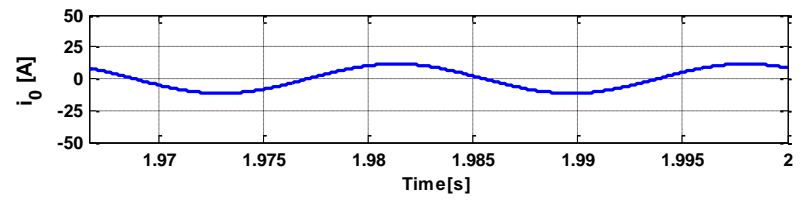

Fig. 3. Measured PCC voltages and currents and resulting components for Case I $-p q$-Theory.

In this case the measured voltages and currents from $F B D$ are practically in-phase, since the return conductor ensures that the load central point matches the source central point (Fig. 2 - left side top). Indeed, it is applicable as long as the impedance of the return conductor were small. In such a case, the measured voltages to the virtual star point, which represents the source central point, will also reflect the load phase voltages and the return to virtual star point voltage will be practically zero $\left(v_{n *(F B D)} \cong 0\right)$. For the same reason, the decomposed active currents yields in-phase and have the same waveform of the voltages $\left(i_{a \mu(F B D)}\right)$, excepted for the neutral active current $\left(i_{a n(F B D)} \cong 0\right)$, as shown in Fig. 2 .
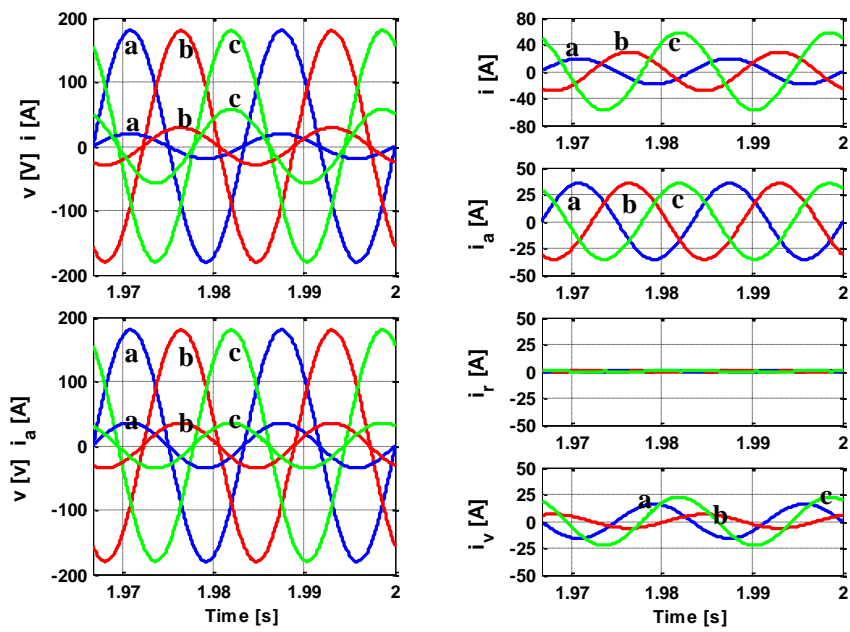

Fig. 4: Measured PCC voltages and currents and resulting components for Case I - CPT-method.

From Figs. 2-4 it is possible to observe that the active currents based on the $F B D$ and $C P T$ methods are equal to the average part of the Akagi's active current $\left(i_{a \mu(C P T)}=\right.$ $\left.i_{a \mu(F B D)}=i_{\bar{p} \mu(p q)}\right)$.

Regarding to the FBD-method, Fig. 2 shows that the neutral active and variation currents are null $\left(i_{\text {an }(F B D)}=\right.$ $\left.i_{v n(F B D)}=0\right)$, however, the powerless current results equal to the original neutral current $\left(i_{z n(F B D)}=i_{n}\right)$. This fact indicates that the consideration of the return conductor as a homogeneous phase conductor should be dealt with very much attention, since the information related to it is very different from the other conductors.

On the other hand, the parcels of powerless current $\left(i_{z \mu(F B D)}\right)$, variation current $\left(i_{v \mu(F B D)}\right)$, oscillating active current $\left(i_{\tilde{p} \mu(p q)}\right)$ and reactive current $\left(i_{q \mu(p q)}\right)$ indicate a third order harmonic content, which is not expected in case of linear resistive loads. Besides, the $F B D$ variation current is equal to the $p q$-Theory oscillating active current $\left(i_{v \mu(F B D)}=\right.$ $\left.i_{\tilde{p} \mu(p q)}\right)$, but the powerless current is not equal to the reactive current $\left(i_{z \mu(F B D)} \neq i_{q \mu(p q)}\right)$. In order to compare and find the 
similarities between $F B D$ and Akagi's methods it is possible to demonstrate that:

$$
i_{z \mu(F B D)}=i_{q \mu(p q)}+i_{0 \mu(p q)}, i_{v \mu(F B D)}=i_{\tilde{p} \mu(p q)}
$$

It can be concluded from Fig. 4 that the $C P T$ approach seems to be more suitable than the other two methods for the analysis of Case I. In such method, the load unbalance is reflected just in the sinusoidal void current component $\left(i_{v \mu(C P T)}\right)$ and the reactive component results null $\left(i_{r \mu(C P T)}=\right.$ $0)$, since there are not energy storage elements on the circuit. Thus, the power phenomena seem to be interpreted more coherently with the traditional concepts.

Because of the lack of additional space, the spectra of the current components were omitted. However, based on their analysis, the following associations may be summarized:

$$
\begin{gathered}
i_{a \mu(F B D)}=i_{\bar{p} \mu(p q)} ; \quad i_{v \mu(F B D)}=i_{\tilde{p} \mu(p q)} ; \\
i_{z \mu(F B D)}=i_{q \mu(p q)}+i_{0 \mu(p q)} ; \\
i_{a n(F B D)}=i_{v n(F B D)}=0 ; \\
i_{z n(F B D)}=i_{n} ; \\
i_{v \mu(C P T)}=i_{z \mu(F B D)}+i_{v \mu(F B D)}=i_{\tilde{p} \mu(p q)}+i_{q \mu(p q)}+i_{0 \mu(p q)} \\
i_{a \mu(C P T)}+i_{v \mu(C P T)}=i_{\mu} ; \\
i_{a \mu(F B D)}+i_{z \mu(F B D)}+i_{v \mu(F B D)}=i_{\mu} ; \\
i_{\bar{p} \mu(p q)}+i_{\tilde{p} \mu(p q)}+i_{q \mu(p q)}+i_{0 \mu(p q)}=i_{\mu}
\end{gathered}
$$

\section{B. Case II - Two non linear and one linear load - small line impedance}

Considering the same power source and line impedances of the Case I, Fig. 5 shows the power circuit for Case II, while Table 2 presents the different load impedances.

The comparisons of the waveforms of Figs. 6-8 and their spectra results in very similar conclusions to those at the end of last section, expect that in this case, the presence of energy

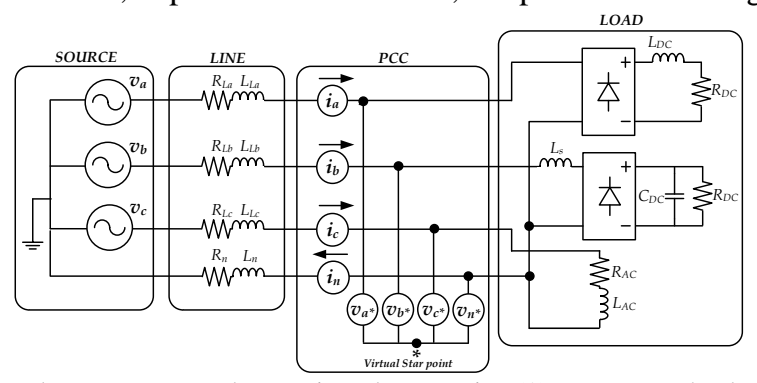

a) Voltages measured to a virtual star point (*): $F B D$-Method.

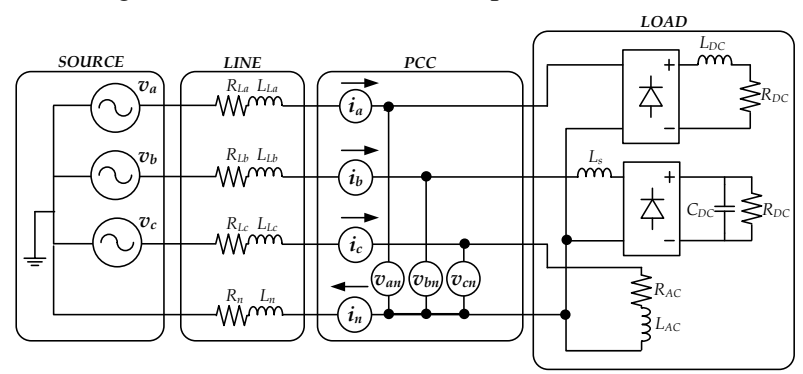

b) Voltages measured to the return conductor: $p q$-Theory and $C P T$.

Fig. 5: Power circuit for Case II - unbalanced non linear load.

\section{TABLE II}

Load impedances for Case II.

$\begin{array}{ccc}\text { RL Rectifier } & \text { RC Rectifier } & \text { RL } \\ =8 \mathrm{mH} \mathrm{R} \mathrm{R}_{\mathrm{DC}}=5 \Omega & \mathrm{C}_{\mathrm{DC}}=8 \mathrm{~m} \Omega \mathrm{R}_{\mathrm{DC}}=4 \Omega & \mathrm{L}_{\mathrm{AC}}=8 \mathrm{mH} \mathrm{R} \mathrm{R}_{\mathrm{AC}}=5 \Omega\end{array}$

storage elements leads to the occurrence of reactive current from the $C P T$-method $\left(i_{r \mu(C P T)} \neq 0\right)$. Observe that while the line impedance were small, the voltage distortion will be small, as well as in the components: $i_{a \mu(C P T)}, i_{r \mu(C P T)}$, $i_{a \mu(F B D)}, i_{\bar{p} \mu(p q)}$. Due to the occurrence of reactive current, in this case the following relationship is applicable:

$$
\begin{aligned}
i_{r \mu(C P T)}+ & i_{v \mu(C P T)}=i_{z \mu(F B D)}+i_{v \mu(F B D)} \\
= & i_{\tilde{p} \mu(p q)}+i_{q \mu(p q)}+i_{0 \mu(p q)} .
\end{aligned}
$$

Note that the $C P T$-method isolates the reactive component $\left(i_{r \mu(C P T)}\right)$ related to the average equivalent susceptance of the circuit, from the void current $\left(i_{v \mu(C P T)}\right)$, related to load unbalances and nonlinearities.

The following relations are also valid:

$$
\begin{aligned}
& i_{a \mu(C P T)}=i_{a \mu(F B D)}=i_{\bar{p} \mu(p q)} \\
& i_{v \mu(F B D)}=i_{\tilde{p} \mu(p q)} \\
& i_{z \mu(F B D)}=i_{q \mu(p q)}+i_{0 \mu(p q)}
\end{aligned}
$$
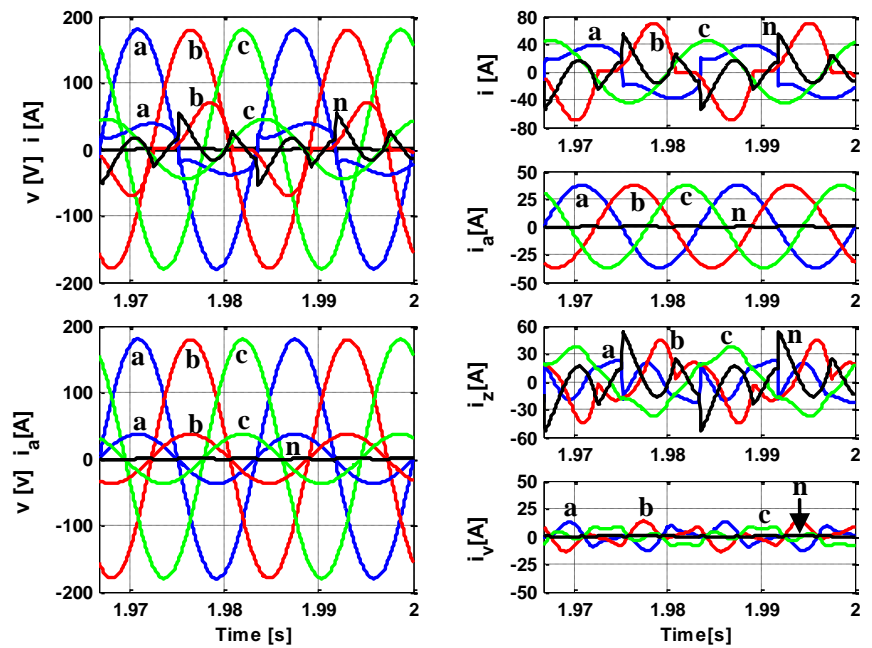

Fig. 6: Measured PCC voltages and currents and resulting components for Case II - FBD-Method.
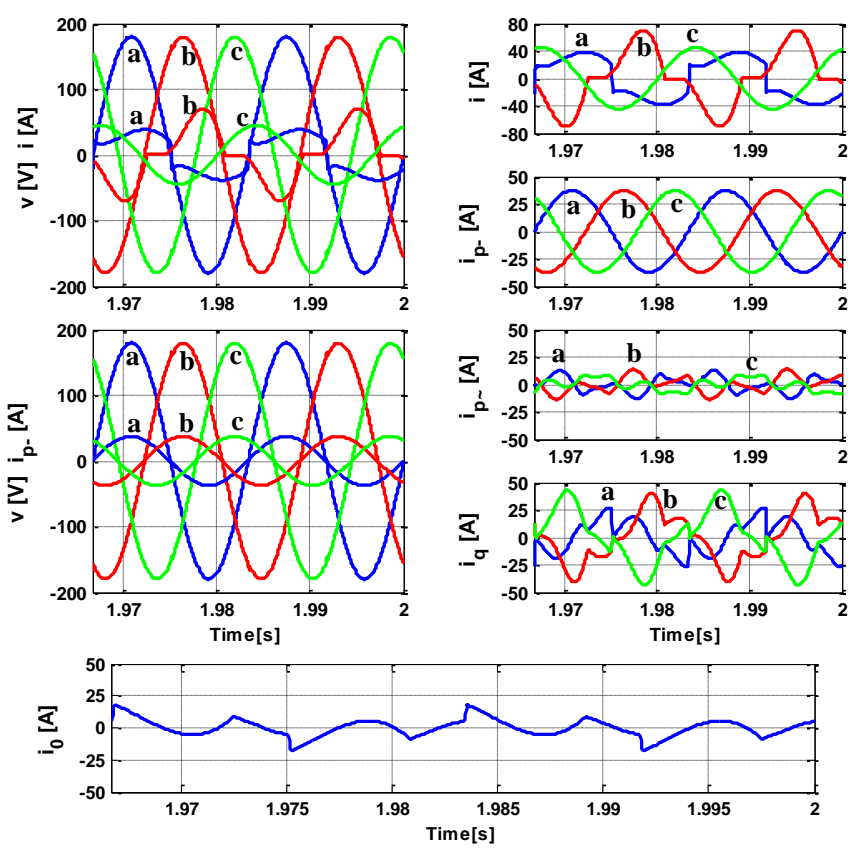

Fig. 7: Measured PCC voltages and currents and resulting components for Case II $-p q$-Theory. 

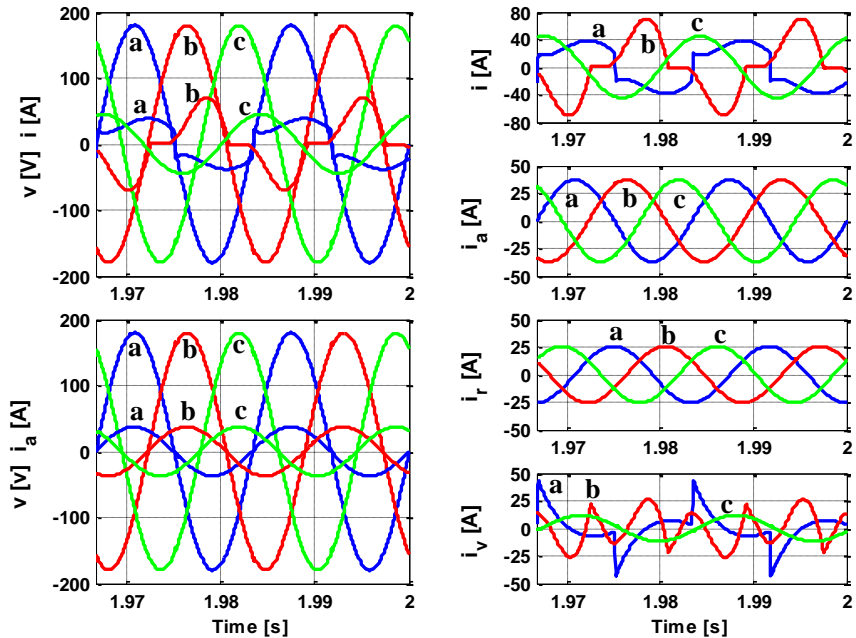

Fig. 8: Measured PCC voltages and currents and resulting components for Case II - CPT-method.

$$
\begin{gathered}
i_{a \mu(C P T)}+i_{r \mu(C P T)}+i_{v \mu(C P T)}=i_{a \mu(F B D)}+i_{z \mu(F B D)}+ \\
i_{v \mu(F B D)}=i_{\bar{p} \mu(p q)}+i_{\tilde{p} \mu(p q)}+i_{q \mu(p q)}+i_{0 \mu(p q)}=i_{\mu} \\
i_{a n(F B D)}=i_{v n(F B D)}=0 ; \\
i_{z n(F B D)}=i_{n} .
\end{gathered}
$$

\section{Case III - Two non linear and one linear load - high line} impedance

Assuming the same circuit of Fig. 5, but changing the line impedance to $R_{L}=10 m \Omega$ and $L_{L}=2 m H$, which represents a week PCC condition; Figs. 9-11 show the resulting current components for each method.

Note that in this case, the voltages are significantly distorted and unbalanced, because of the larger line impedance. This was not observed in the previous two cases. Besides, based on the distinct voltage referential, the $F B D$ voltages do not coincide with $p q$ and $C P T$, which are equal.

Based on the Blakesley Theorem [40], the authors have discussed in details the influence of such referential [32], and it is possible to conclude that from the point of view of power measurements or calculation, both can be applied (virtual star point or return conductor). However, very much attention should be considered if one is interested on the resulting current components for physical phenomena interpretation, power quality monitoring or even to power conditioning. In this case:

$$
\begin{aligned}
& v_{\mu n} \neq v_{\mu *}, \\
& v_{n *} \neq 0,
\end{aligned}
$$

what leads to the following conclusions:

- The $F B D$ active currents $\left(i_{a \mu(F B D)}\right)$ are in-phase with and have the same waveform of the respective voltages to the star point $\left(v_{\mu *}\right)$;

- The $C P T$ active currents $\left(i_{a \mu(C P T)}\right)$ are in-phase with and have the same waveform of the respective voltages to the return (neutral) conductor $\left(v_{\mu n}\right)$;

- The average parts of the active currents from the $p q$ Theory are in-phase with the voltages to the neutral conductor $\left(v_{\mu n}\right)$, but they have not the same waveform.

Thus, different from all previous cases, here it is demonstrated that under such conditions, even the $F B D$ and $C P T$ active currents may not match:

$$
i_{a \mu(C P T)} \neq i_{a \mu(F B D)} \text {. }
$$

But one could ask why, since both are based on the Fryze's definition? The answer regards to the fact that the virtual referential reflects the voltages from the PCC to the source central point, including the voltage drops across the line impedances. On the other hand, the neutral referential reflects the voltages from the PCC to the load central point or the voltages over the phase equivalent load impedances.

Considering the Akagi's average active currents $\left(i_{\bar{p} \mu(p q)}\right)$, they have not the same waveform of the voltages, since the ratios on equation (21) are not constants. Such ratio will be constant only in case of balanced and sinusoidal voltages.
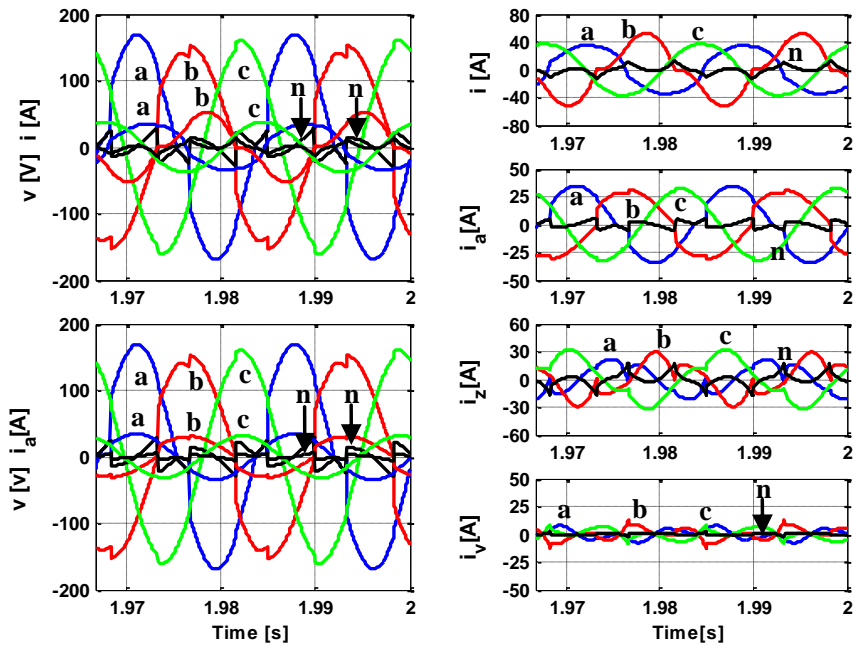

Fig. 9: Measured PCC voltages and currents and resulting components for Case III - FBD-Method.
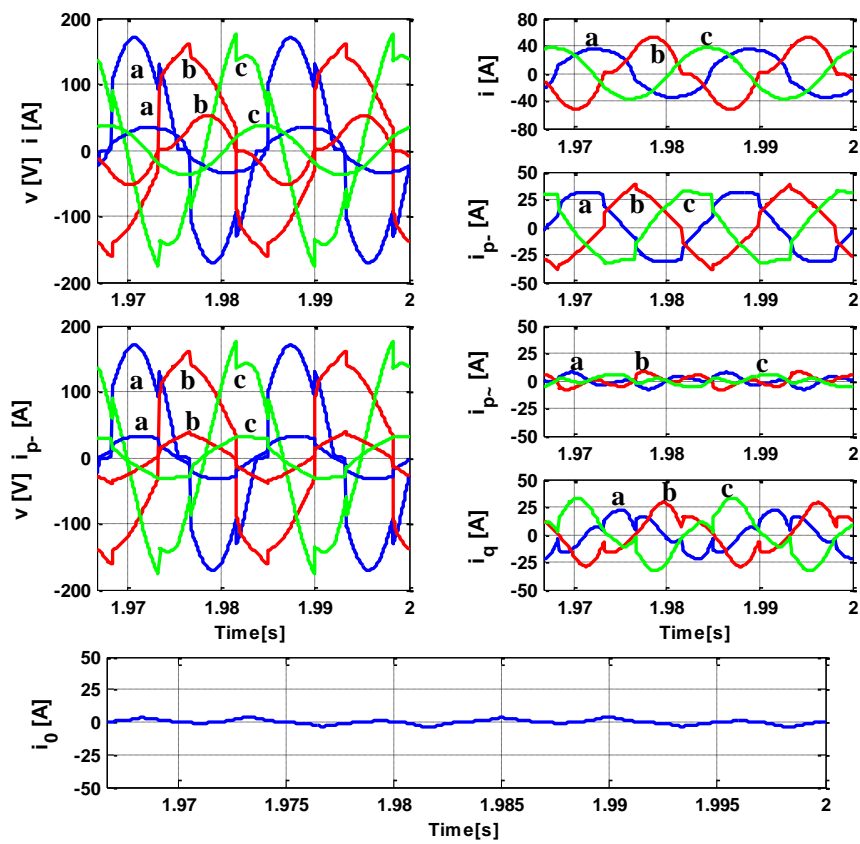

Fig. 10: Measured PCC voltages and currents and resulting components for Case III $-p q$-Theory. 
Thus, the active currents from the three methods have no relation in this case:

$$
i_{a \mu(C P T)} \neq i_{a \mu(F B D)} \neq i_{\bar{p} \mu(p q)} .
$$

Regarding to the differences between the $F B D$ and $C P T$ active currents, one could apply the correction method proposed in [32], which consist in adjusting the voltages measured using the virtual star point, so that they match the voltages measured to the return conductor, on the load side.

From the waveforms of Figs. 9-11 and the respective spectra, it is also possible to conclude that:

$$
\begin{aligned}
& i_{z \mu(F B D)} \neq i_{q \mu(p q)} ; \quad i_{v \mu(F B D)} \neq i_{\tilde{p} \mu(p q)} ; \\
& i_{z \mu(F B D)} \neq i_{q \mu(p q)}+i_{0 \mu(p q)} ; \\
& i_{a \mu(C P T)}+i_{r \mu(C P T)}+i_{v \mu(C P T)}=i_{\mu} ; \\
& i_{0 \mu(p q)}+i_{\bar{p} \mu(p q)}+i_{\tilde{p} \mu(p q)}+i_{q \mu(p q)}=i_{\mu} ; \\
& i_{a \mu(F B D)}+i_{z \mu(F B D)}+i_{v \mu(F B D)}=i_{\mu} ; \\
& i_{n}=i_{a n(F B D)}+i_{z n(F B D)}+i_{v n(F B D)} ; \\
& i_{r \mu(C P T)}+i_{v \mu(C P T)} \neq i_{z \mu(F B D)}+i_{v \mu(F B D)} \\
& \quad \neq i_{\tilde{p} \mu(p q)}+i_{q \mu(p q)}+i_{0 \mu(p q)} .
\end{aligned}
$$
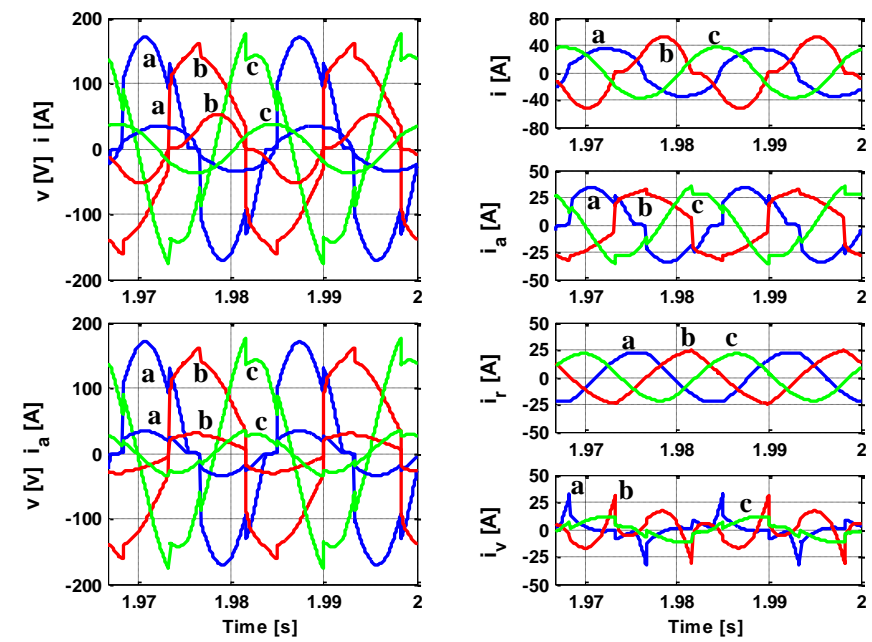

Fig. 11: Measured PCC voltages and currents and resulting components for Case III - CPT method.

\section{CONCLUSIONS}

Apart from any discrepancy in case of three-phase threewire circuits [31], the examples proposed in this paper demonstrate that the differences among the resulting current components can be even bigger for four-wire circuits. Particularly in Case III, the voltage referential has great influence on the results, as a function of the weak PCC.

It was possible to observe that the physical phenomena interpretation is not an easy assignment under such conditions. As a whole, the $C P T$ proposal seems to be a suitable approach, since the resulting active currents represent the currents drawn by a balanced equivalent resistive load and the reactive currents represent the currents drawn by a balanced equivalent reactance. The residual components (the void currents) represent the load unbalances and the nonlinearities of the power circuit.

As a matter of fact, the conclusions of this paper and related work lead these authors to conclude that the point of defining a novel general applicable power theory and related current decompositions, which has been motivated in the last decades based on the increasing use of non linear and unbalanced load, has been boosted up in the last few years based on the novel configurations of modern power grids, especially those with relatively low short circuit levels (such as those related to microgrids).

These authors are now working on how to split the influence of unbalances and harmonic distortions on the $C P T$ void current, as well as, evaluating the need of identifying or not fundamental $(60 / 50 \mathrm{~Hz})$ current components, as proposed by [5]. Future papers will deal with these questions.

\section{REFERENCES}

[1] C. I. Budeanu, "Puissances Reactives et Fictives" Instytut Romain de l'Energie, Pub. no. 2, Bucharest, Rumania, 1927.

[2] N. L. Kuster and W. J. More, "On the Definition of Reactive Power under Non-Sinusoidal Condition", IEEE Trans. on Power Apparatus and Systems, vol. PAS-99, no. 5, pp. 1845-1854, Sept/Oct 1980.

[3] W. Shepherd, P. Zakikhani "Suggested Definition of Reactive Power for Non-sinusoidal Systems", Proc. Inst. Elec. Eng., vol. 119, pp. 1361-1362, Sept. 1972.

[4] L. S. Czarnecki, "Orthogonal Decomposition of the Currents in a 3-Phase Nonlinear Asymmetrical Circuit with a Nonsinusoidal Voltage Source", IEEE Trans. on Instrumentation and Measurements, vol. 37, no. 1, pp. 30-34, March 1988.

[5] IEEE Trial-Use Standard Definitions for the Measurement of Electric Power Quantities under Sinusoidal, Non-sinusoidal, Balanced or Unbalanced Conditions, IEEE Standard 1459-2000, June, 2000.

[6] L.S. Czarnecki, “Currents' Physical Components (CPC) in Circuits with Non-sinusoidal Voltages and Currents. Part 1: Single-phase linear circuits", Electrical Power Quality and Utilization Journal, vol. XI, no. 2, pp. 3-14, 2005.

[7] L.S. Czarnecki, "Currents Physical Components (CPC) in Circuits with Non-sinusoidal Voltages and Currents. Part 2: Three-phase three-wire linear circuits," Electrical Power Quality and Utilization Journal, vol. XII, no. 1, pp. 3-13, 2006.

[8] S. Fryze, "Wirk-, Blind- und Scheinleistung in Elektrischen Stromkreisen mit Nichtsinusförmigem Verlauf von Strom und Spannung", Elektrotechnische Zeitschrift, vol. 53, no. 25, pp. 596-599, 625-627, 700702, 1933 .

[9] F. Buchholz, Das Begriffsystem Rechtleistung, Wirkleistung, totale Blindleistung, Selbstverlag München, 1950.

[10]M. Depenbrock, "The FBD-Method, a Generally Applicable Tool For Analyzing Power Relations", IEEE Trans. on Power Systems, vol. 8, no. 2, 381-387, May 1993.

[11]M. Depenbrock, Quantities of a Multiterminal Circuit Determined on the Basis of Kirchhoff's Laws, ETEP European Trans. on Electrical Power Engineering , vol. 8, no. 4, pp. 249- 257, 1998.

[12]H. Akagi, Y. Kanazawa, A. Nabae, "Generalized Theory of the Instantaneous Reactive Power in Three- 
Phase Circuits", in Proc. of International Power Electronics Conference JIEE-IPEC, pp. 1375-1386, 1983.

[13] H. Akagi, A. Nabae, The p-q Theory in Three-Phase Systems under Non-Sinusoidal Conditions", ETEP European Trans. on Electrical Power Engineering, vol. 3, no. 1, pp. 27-31, Jan./Feb. 1993.

[14]P. Tenti and P. Mattavelli, “A Time-Domain Approach to Power Term Definitions under Non-sinusoidal Conditions", in Proc. of Sixth International Workshop on Power Definitions and Measurements under NonSinusoidal Conditions, 2003.

[15] P. Tenti, E. Tedeschi, P. Mattavelli, “Optimization of Hybrid Filters for Distributed Harmonic and Reactive Compensation", in Proc. of IEEE International Conference on Power Electronics and Drive Systems PEDS, vol. 01, pp. 292-297, 2005.

[16]F. Peng, G. Ott, and D. Adams, "Harmonic and Reactive Power Compensation Based on the Generalized Instantaneous Reactive Power Theory for 3-phase 4-wire Systems", in Proc. of IEEE Power Electronics Specialists Conference PESC, vol. 2, pp. 1089-1095, 1997.

[17] J. L. Willems, "Instantaneous Sinusoidal and Harmonic Active and Deactive Currents in Threephase Power Systems", ETEP European Trans. on Electrical Power Engineering, vol. 4, no. 5, pp. 335343. 1994.

[18]F. P. Marafão, Analysis and Control of Electrical Energy by means of Digital Processing Techniques. PhD Thesis. University of Campinas, Brazil, 2004. (in Portuguese).

[19]E. Watanabe and M. Aredes, "Compensation of Nonperiodic Currents Using the Instantaneous Reactive Power Theory", in Proc of Power Eng. Soc. Summer Meeting, vol. 2, pp. 994-999, 2000.

[20] M. K. Mishra, A. Joshi, and A. Ghosh, "Unified Shunt Compensator Algorithm Based on Generalized Instantaneous Reactive Power Theory," IEEE Trans. Gen, Trans, Distrib., vol. 148, no. 6, pp. 583-589, Nov. 2001.

[21]H. Kim, F. Blaabjerg, B. Bak-Jensen, and J. Choi, "Instantaneous Power Compensation in Three-phase Systems by Using p-q-r Theory", IEEE Trans. Power Electronics, vol. 17, no. 5, pp. 701-710, Sep. 2002.

[22] E. H. Watanabe, R. M. Stephan, and M. Aredes, "New Concept of Instantaneous Active and Reactive Powers in Electrical Systems with Generic Loads", IEEE Trans. Power Delivery vol. 8, no. 2, pp. 697-703, Apr. 1993.

[23] H. Kim, F. Blaabjerg, and B. Bak-Jensen, "Spectral Analysis of Instantaneous Powers in Single-phase and Three-phase Systems with use of p-q-r Theory". IEEE Transaction on Power Electronics vol. 17, no. 5, pp. 711-720, Sep. 2002.

[24] W. G. Morsi, and M. E. El-Hawary, "Defining Power Components in Nonsinusoidal Unbalanced Polyphase Systems: The Issues", IEEE Trans. Power Delivery, vol. 22, no. 4, pp. 2428-2438, October 2007.
[25]R. S. Herrera and P. Salmerón, "Instantaneous Reactive Power Theory: A Comparative Evaluation of Different Formulations", IEEE Trans. Power Delivery, vol. 22, no. 1, pp. 595-604, Jan. 2007.

[26] M. Malengret and C. T. Gaunt, "Decomposition of Currents in Three- and Four-Wire Systems", IEEE Trans. on Instrumentation and Measurement, vol. 57, no. 5, pp. 963-972, May 2008.

[27] J. L.Willems, "A New Interpretation of the AkagiNabae Power Components for Nonsinusoidal Threephase Situations," IEEE Trans. on Instrumentation and Measurement, vol. 41, no. 4, pp. 523-527, Aug. 1992.

[28] M. Depenbrock, V. Staudt and H. Wrede, "Theoretical Investigation of Original and Modified Instantaneous Power Theory Applied to Four-Wire Systems". IEEE Trans. on Industrial Application, vol. 39, no. 4, pp. 1160-1168, July/Aug 2003.

[29] L. S. Czarnecki, "On Some Misinterpretations of the Instantaneous Reactive Power p-q Theory", IEEE Trans. Power Electronics vol. 19, no. 3, pp. 828-836, May. 2004.

[30]L. S. Czarnecki, "Instantaneous Reactive Power p-q Theory and Power Properties of Three-phase Systems," IEEE Trans. Power Delivery, vol. 21, no. 1, pp. 362-367, Jan. 2006.

[31]H. K. M. Paredes, F. P. Marafão, L. C. P. da Silva, “A Comparative Analysis of FBD, PQ and CPT Current Decompositions - Part I: Three-Phase Three-Wire Systems", in Proc. of IEEE PowerTech, 2009.

[32]F. P Marafão, S. M. Deckmann, and H. K. Morales P. "The influence of the Voltage Referential to Power Quality Evaluation", IEEE Latin America Magazine, vol. 6, no. 1, PP. 81-88, March 2008. (in Portuguese).

[33]E. Tedeschi and P. Tenti, "Cooperative Design and Control of Distributed Harmonic and Reactive Compensators", in Proc. of International School on Nonsinusoidal Currents and Compensation (ISNCC), pp. 1-6, June 2008.

[34] A. Blondel, "Measurement of Energy of Polyphase currents", in Proc. of International Electrical Congress, pp. 112-117, 1893.

[35] A. E. Emanuel, "Reflections on the Effective Voltage Concept", in Proc. of International Workshop on Power Definitions and Measurements under Nonsinusoidal Conditions, 2003.

[36] J. L. Willems and J. A. Ghijselen, "The Choice of the Voltage Reference and the Generalization of the Apparent Power", in Proc. of International Workshop on Power Definitions and Measurements under Nonsinusoidal Conditions, 2003.

[37] J. L. Willems, J. A. Ghijselen and A. E. Emanuel, "The Apparent Power Concept and the IEEE Standard 1459-2000", IEEE Trans. Power Delivery, vol. 20, no. 2, pp. 876-884, Apr. 2005.

[38] J. Willems, "Critical Analysis of Different Current Decomposition and Compensation Schemes", in Proc. of International School on Nonsinusoidal Currents and

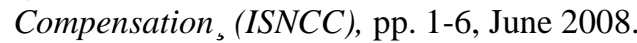


[39] E. Clarke, Circuit Analysis of A-C power systems, vol. I - Symmetrical and Related Components, New York: John Wiley and Sons, Inc, 1943.

[40]T. H. Blakesley, "A New Electrical Theorem", in Proc. of the Physical Society of London, vol.13, pp. 65-67, 1894.

[41] V. Staudt, "Fryze - Buchholz - Depenbrock: A TimeDomain Power Theory", in Proc. of International School on Nonsinusoidal Currents and Compensation, (ISNCC), pp. 1-6, June 2008.

\section{BIOGRAPHIES}

Fernando Pinhabel Marafão was born in José Bonifácio, Brazil in 1975. He received his B.S. degree (1998) in Electrical Engineering from Unesp - Univ Estadual Paulista, Brazil. In 2000 and 2004 he received, respectively, his Master and $\mathrm{PhD}$ degrees from University of Campinas, Brazil. His current interests are mainly concerned with different power definitions under multiphase nonlinear systems and its applications in power conditioning and revenue metering.

He held in 2002, a visitor student position at the Department of Information Engineering of the University of Padova (Italy), working on digital control techniques for active power filters. In 2005 he joined the Group of Automation and Integrating Systems at Unesp, Sorocaba, Brazil, where he has been an Assistant Professor. Since 2007, he is also an external Professor of the Electrical Engineering Department of the Campus of Bauru (Unesp).

Dr. Marafão is a member of the Brazilian Power Electronics Society (SOBRAEP), Brazilian Automatic Society (SBA) and IEEE.

Helmo Kelis Morales Paredes was born in Puno-Perú. He received his B.S. degree (2002) in Electrical Engineering from Arequipa National University (UNSA), Perú. In 2006 he received his Master degrees from University of Campinas (UNICAMP), Brazil. Currently, he is a PhD student at UNICAMP. His current interests are mainly concerned with power quality evaluation and power definitions under multiphase nonlinear systems.

MSc. Paredes is a member of the Brazilian Power Electronics Society (SOBRAEP), and IEEE.

Luiz Carlos Pereira da Silva was born in Brazil in 1972. He graduated in electrical engineering from the Federal University of Goiás, Brazil in 1995, and obtained MSc and $\mathrm{PhD}$ degrees in power systems engineering from the University of Campinas, Brazil, in 1997 and 2001 respectively.

He worked as a visiting researcher at the University of Alberta, Canada, in 1999-2000, and as a guest professor at the Technical University of Denmark, in 2008. Since 2002 he is with the University of Campinas, Brazil, where he is an Associate Professor. His main areas of interest are power system stability and control and distributed generation. 